\title{
Combination of azathioprine and UVA irradiation is a major source of cellular 8-oxo-7,8-dihydro-2'-deoxyguanosine
}

\author{
Marcus S. Cooke ${ }^{* 1}$, Tiago L. Duarte ${ }^{2}$, Deborah Cooper, Jie Chen, \\ Sridevi Nandagopal and Mark D. Evans
}

Radiation and Oxidative Stress Group, Dept. Cancer Studies and Molecular Medicine, *\& Dept. Genetics, Robert Kilpatrick Clinical Sciences Building, University of Leicester, Leicester, LE2 7LX, UK.

Running title: azathioprine + UVA = 8-oxodG

Key words: azathioprine, oxidative stress, UV radiation, 8-oxo-7,8-dihydro-2'deoxyguanosine (8-oxodG), comet assay.

\footnotetext{
${ }^{1}$ To whom correspondence should be addressed. Dr. Marcus S. Cooke, Radiation and Oxidative Stress Group, Dept. Cancer Studies and Molecular Medicine, \& Dept. Genetics, Robert Kilpatrick Clinical Sciences Building, University of Leicester, Leicester, LE2 7LX, UK. +44 (0) 116 2525825, msc5@le.ac.uk.

${ }^{2}$ Present address: Iron Genes and Immune System Laboratory, Instituto de Biologia Molecular e Celular, Universidade do Porto, Portugal
} 


\section{ABSTRACT}

Thiopurine antimetabolites, such as azathioprine (Aza) and 6-thioguanine (6-TG), are widely used in the treatment of cancer, inflammatory conditions and organ transplantation patients. Recent work has shown that cells treated with 6-TG and UVA generate ROS, with implied oxidatively generated modification of DNA. In a study of urinary 8-oxo-7,8-dihydro-2'-deoxyguanosine (8-oxodG) in renal transplant patients, we provided the first in vivo evidence linking Aza and oxidatively damaged DNA. Using the hOGG1 comet assay we herein demonstrate high levels of 8-oxodG and alkali-labile sites (ALS) in cells treated with biologically relevant doses of 6-TG, or Aza, plus UVA. This damage was induced dose-dependently. Surprisingly, given the involvement of 6-TG incorporation into DNA in its therapeutic effect, significant amounts of 8-oxodG and ALS were induced in quiescent cells, although less than in proliferating cells. We speculate that some activity of hOGG1 towards unirradiated, 6-TG treated cells, implies possible recognition of 6-TG or derivatives thereof. This is the first report to conclusively demonstrate oxidatively damaged DNA in cells treated with thiopurines and UVA. These data indicate that Aza-derived oxidative stress will occur in the skin of patients on Aza, following even low level UVA exposure. This is a probable contributor to the increased risk of non-melanoma skin cancer in these patients. However, as oxidative stress is unlikely to be involved in the therapeutic effects of Aza, intercepting ROS production in the skin could be a viable route by which this side effect may be minimised. 


\section{Introduction}

The thiopurine antimetabolites, such as azathioprine (Aza) and 6-thioguanine (6-TG), are widely used in the treatment of cancer and inflammatory conditions [1], as well as in the therapy of organ transplant patients [2]. However, despite there being a significant amount of literature concerning the use of thiopurines, little is known concerning their mode of action [3]. It is clear that the inactive pro-drugs, 6-TG or Aza, are metabolised to DNA precursors, such as 6-thio-2'-deoxyguanosine triphosphate (6-TdGTP) [4], in the 2'-deoxyribonucleotide pool where they are substrates for DNA polymerases. Incorporation into DNA appears to be, in part, necessary for their cytotoxic action [4,5], leading to a variety of DNA modifications, such as chromatid damage [6], DNA strand breaks and DNA-protein crosslinks. Also required is an active DNA mis-match repair system, and whilst the effects of purine starvation have been suggested, DNA damage seems to be the main mechanism for the cytotoxic effects of thiopurines [7].

It is well established that transplant patients are at a high (some 50- to 200fold [8]) risk of developing malignancy, most frequently non-melanoma skin cancer $[9,10]$, and squamous cell carcinoma (SCC) specifically, which has been largely attributed to immunosuppression, in conjunction with ultraviolet radiation (UVR) exposure [11]. Indeed Aza, specifically, has been shown to enhance UV-induced skin carcinogenesis in mice [12]. The carcinogenic effect of UVR is therefore particularly relevant to transplant patients. Although the predominant DNA modifications induced by solar UVR are dimeric photoproducts, such as cyclobutane pyrimidine dimers (CPD [13]), UVR also generate reactive oxygen species (ROS), which can lead to the oxidatively generated modification of various cellular molecules, including DNA [14]. The ROS produced by UVA is predominantly singlet oxygen $\left({ }^{1} \mathrm{O}_{2}\right)$ [15], 
albeit with some contribution from hydroxyl radicals $\left({ }^{\bullet} \mathrm{OH}\right)$ [14], whereas the mechanism for UVB-induced formation of oxidatively modified DNA is less clear, and may involve formation of, oxygen independent, nucleobase radical cations, ${ }^{\bullet} \mathrm{OH}$ and ${ }^{1} \mathrm{O}_{2}$ [14], or combinations thereof. ROS-induced DNA damage is of particular relevance to carcinogenesis [16], as over 70 products have been described [17], many of which have proven cellular effects related to carcinogenesis [18]. The induction of oxidatively generated damage to DNA is therefore likely to be an important factor in UVR-induced carcinogenesis [19-22].

Recent work has shown that incorporation of 6-TG into DNA of cultured cells, and subsequent exposure to UVA generates ROS, with implied oxidatively generated modification of DNA [23]. This study was followed up with a report which provided evidence for the formation of novel DNA lesions, of which guanine-6-sulphonate $\left(\mathrm{G}^{\mathrm{SO}}\right)$ appeared to predominate, following UVA irradiation of free 6-TG and 6-TGcontaining oligomers, and that singlet oxygen was most likely to be the ROS involved [24]. The induction of oxidative stress may well contribute towards the phototoxicity and lowering of minimal erythemal dose for UVA irradiation, following treatment of mice [25] or humans [23] respectively, with Aza.

We recently described a study of urinary 8-oxo-7,8-dihydro-2'deoxyguanosine (8-oxodG), a widely used biomarker of oxidative stress, in renal transplant patients with and without SCC [26]. In addition to providing evidence that a sub-population of renal transplant patients are under greater oxidative burden, and are particularly predisposed to skin cancer, we also noted a significant association between Aza treatment and urinary 8-oxodG $(P=0.02)$, providing the first in vivo evidence linking Aza use and oxidatively modified DNA. The purpose of the present study was to investigate, in vitro, whether azathioprine, in conjunction with UVA, 
may generate significant amounts of oxidatively damaged DNA (8-oxodG and alkalilabile sites, ALS), under physiological conditions and at biologically relevant doses. 


\section{Materials and methods}

\subsection{Reagents}

Minimal essential medium (MEM) with Earle's salts and non-essential amino acids, fetal bovine serum (FBS), and Glutamax-I were purchased from Invitrogen (Paisley, UK). The DNA repair enzyme, human 8-oxoguanine DNA glycosylase 1 (hOGG1), was purchased from New England Biolabs (Hitchin, UK). Aza and 6-TG, along with all other chemicals and reagents were purchased from Sigma-Aldrich (Poole, UK), unless otherwise stated.

\subsection{Cell line and culture conditions}

GM5399 primary human diploid fibroblasts (HDFs), whose provenance is described elsewhere [27], were purchased from NIGMS Human Genetic Cell Repository (Coriell Institute for Medical Research, Camden, NJ). Cells were grown as a monolayer in MEM with Earle's salts, supplemented with $2 \mathrm{mM}$ Glutamax-I, nonessential amino acids, $10 \%(\mathrm{v} / \mathrm{v}) \mathrm{FBS}$, at $37{ }^{\circ} \mathrm{C}$, in a humidified atmosphere, comprising $5 \% \mathrm{CO}_{2}$. In experiments where serum starvation was performed, cells were grown until nearly confluent and subsequently incubated in medium containing $0.5 \%$ FBS for further $48 \mathrm{~h}$.

\subsection{Azathioprine/6-thioguanine treatment and UVA irradiation}

Sub-confluent cells were incubated with clinically relevant doses of either 6-TG or Aza $(1 \mu \mathrm{M}$, final concentration, or in the case of the dosing experiments $0.2 \mu \mathrm{M}, 1$ $\mu \mathrm{M}, 5 \mu \mathrm{M}$ 6-TG [28]) for $48 \mathrm{~h}$, prior to irradiation with $1 \mathrm{~J} / \mathrm{cm}^{2}$ UVA. UVA irradiation was performed using a custom-made exposure cabinet (Hybec Ltd, 
Leicester, UK), which contains a bank of six Philips Cleo Perfomance/40 W fluorescent tubes, with a Schitt Desag M-UG2 UV transmitting absorption glass filter (HV Skan, Solihull, UK) to remove both visible and infrared wavelengths. The wavelength emission spectrum of this filtered source was characterised using a single monochromator diode array spectrometer, and confirmed to be free of UVB (Laboratory of Atmospheric Chemistry, Department of Chemistry, University of Leicester). Dosimetry was determined using an MP-100 UV radiometer, in conjunction with an MP-136 UV sensor (Knight Optical Technologies, Surrey, UK). Culture medium was removed and the cells rinsed with PBS prior to irradiation, on ice, with the cells covered in a minimal volume of PBS. Immediately following irradiation, cells were prepared for the comet assay, where appropriate, as follows.

2.4. Human 8-oxoguanine glycosylase 1 (hOGG1)-modified comet assay (hOGG1 comet)

DNA damage was assessed using the human 8-oxoguanine glycosylase 1 (hOGG1)modified comet assay (hOGG1 comet), first reported by Smith et al. [29], but with specific refinements of this assay. Both the concentration of hOGG1 enzyme and the incubation period were optimised at $50 \mu \mathrm{L} /$ gel of hOGG1 [diluted 1:500 in enzyme reaction buffer (40 mM HEPES, $0.1 \mathrm{M} \mathrm{KCl}, 0.5 \mathrm{mM}$ disodium EDTA, $0.2 \mathrm{mg} / \mathrm{mL}$ BSA, $\mathrm{Ph} 8.0$ ) to a final concentration of $3.2 \mathrm{U} / \mathrm{mL}]$ and at $37{ }^{\circ} \mathrm{C}$, in a humidified atmosphere, for $45 \mathrm{~min}$. Comet assay conditions were as those described by Duarte et al. [27], as below.

HDFs were suspended in $0.6 \%$ low melting point agarose. Eighty microlitres of the agarose gel (containing approximately $2 \times 10^{4}$ cells) were dispensed onto glass microscope slides, coated previously with $1 \%$ normal melting point agarose. The 
agarose was allowed to set on ice under a coverslip and the slides left overnight in icecold lysis buffer (100 mM disodium EDTA, $2.5 \mathrm{M} \mathrm{NaCl}, 10 \mathrm{mM}$ Tris-HCl, $\mathrm{pH}$ 10, containing $1 \%$ triton X-100 which was added freshly). Slides were washed once with distilled water and immersed in two changes of enzyme digestion buffer [40 mM HEPES, 0.1 M KCl, $0.5 \mathrm{mM}$ EDTA and $0.2 \mathrm{mg} / \mathrm{ml} \mathrm{BSA} \mathrm{(pH} \mathrm{8.0)],} \mathrm{for} 5 \mathrm{~min}$ each time, at room temperature. hOGG1 was added to the gel $(50 \mu \mathrm{L} / \mathrm{gel})$ diluted 1:500 in enzyme reaction buffer to a final concentration of $3.2 \mathrm{U} / \mathrm{mL}$. Gels were covered with a cover slip and incubated in a humidified chamber for $45 \mathrm{~min}$ at $37^{\circ} \mathrm{C}$. The cover slips were removed and the slides were placed in a horizontal electrophoresis tank, covered with cold alkaline electrophoresis buffer $(300 \mathrm{mM} \mathrm{NaOH}, 1 \mathrm{mM}$ disodium EDTA, $\mathrm{pH} \geq 13)$ for $20 \mathrm{~min}$ and electrophoresed at $27 \mathrm{~V}(0.7 \mathrm{~V} / \mathrm{cm})$ and $300 \mathrm{~mA}$ for 20 min. Discrimination between AP sites and SSB was achieved by performing electrophoresis at $\mathrm{pH}>13$ vs. $\mathrm{pH} \sim 12$, respectively [30]. Slides were neutralised with $0.4 \mathrm{M}$ Tris-HCl, pH 7.5 for $20 \mathrm{~min}$, washed with distilled water, and then allowed to dry. All procedures were carried out under subdued light to minimise adventitious DNA damage. For staining, slides were re-hydrated in distilled water, incubated with a freshly made solution of $2.5 \mu \mathrm{g} / \mathrm{mL}$ propidium iodide (PI) for 20 min, washed again for $30 \mathrm{~min}$ and allowed to dry. Comets were visualised by fluorescence microscopy at x200 magnification. Images were captured by an on-line CCD camera and analysed with the Komet Analysis software version 5.5 (Andor Bioimaging, Nottingham, UK). A total of 100 cells were analysed per sample, 50 per duplicate slide. The percentage of DNA in the tail of the comet ( $\%$ tail DNA) was calculated for each cell by the Komet Analysis software. 


\subsection{T4 endonuclease V-modified comet assay (T4 endoV comet)}

Cyclobutane pyrimidine dimers (CPD) were assessed using the T4 endoV cometmodified comet assay [31], with refinements for the cell type used here. Both the concentration of $\mathrm{T} 4 \mathrm{endoV}$ and the incubation period were optimised at $60 \mu \mathrm{L} / \mathrm{gel}$ of T4 endoV [diluted 1:100 in enzyme reaction buffer (40 mM HEPES, 0.1 M KCl, 0.5 $\mathrm{mM}$ disodium EDTA, $0.2 \mathrm{mg} / \mathrm{mL}$ BSA, $\mathrm{pH} 8.0$ ) to a final concentration of $0.1 \mathrm{U} / \mathrm{mL}$ ] and $60 \mathrm{~min}$, at $37^{\circ} \mathrm{C}$ in a humidified atmosphere. Comet assay conditions were as those described by Duarte et al. [27], above.

\subsection{Cell viability}

For the routine assessment of loss of membrane integrity, cells underwent the trypan blue dye exclusion assay. However, for the assessment of cytotoxicity, cells were examined by the PI uptake assay, as described elsewhere [27]. Briefly, cells were preincubated with 6-TG or Aza $(1 \mu \mathrm{M})$ for $48 \mathrm{~h}$, and then irradiated with $1 \mathrm{~J} / \mathrm{cm}^{2} \mathrm{UVA}$, or sham irradiated, as described above. Cells were then replated in fresh medium, in the absence of 6-TG or Aza, and returned to the incubator for $48 \mathrm{~h}$ after which they were resuspended in PBS containing $5 \mu \mathrm{g} / \mathrm{mL}$ PI. A FACScan flow cytometer, in conjunction with Cell Quest software, was used to analyse 10,000 cells per treatment. Identification of dead cells was via a FL2 vs. forward light scatter plot, by their inability to exclude PI and a decrease in cell size. 


\section{Results}

\subsection{Induction of 8-oxodG in cellular DNA}

Initial experiments using the hOGG1 comet demonstrated significant $(\mathrm{P}<0.0001)$ levels of both 8-oxodG and ALS in HDFs treated with $1 \mu \mathrm{M}$ 6-TG in conjunction with $1 \mathrm{~J} / \mathrm{cm}^{2} \mathrm{UVA}$, compared to the corresponding, untreated, sham-irradiated control $(\mathrm{CSI}+6-\mathrm{TG}+\mathrm{hOGG1}$; Figure 1A). Performing comet analysis, in the absence of hOGG1, allows for the examination of single strand breaks (SSB) and, at $\mathrm{pH}>13$, ALS, which may include apurinic/apyrimidinic (AP) sites, oxidised AP sites and certain modified bases [32], depending upon the DNA damaging system used. This demonstrated that approximately $50 \%$ of the damage revealed by hOGG1 comet was ALS, levels of which were also statistically significant $(\mathrm{P}<0.0001)$, compared to the corresponding control (CSI + 6-TG - hOGG1). Consistent with a recent report [13], we noted essentially no 8-oxodG formation in cells treated with UVA alone, likewise we saw no formation of ALS, not least due to the dose used (Figure 1A). Whilst treatment of HDFs with $1 \mu \mathrm{M}$ 6-TG, in the absence of UVA, failed to produce any damage, as determined by the alkaline comet assay; when hOGG1 was employed, significant levels of damage were revealed ( $\mathrm{P}<0.0001$, compared to CSI + hOGG1). A number of reports have indicated that the induction of CPD by UVA is much greater than previously thought $[13,33]$ and hence, could be responsible for the mutagenicity of Aza in conjunction with UVA. However, we noted a minimal increase in CPD, compared to 8-oxodG and ALS (Figure 1A).

Experimentally, 6-TG is frequently used as a surrogate for Aza, to obviate the need for metabolic activation, however, we also examined whether HDFs could metabolise Aza to thioguanine 2'-deoxyribonucleotide triphosphate, and subsequently damage DNA. The results obtained using Aza ( $1 \mu \mathrm{M}$ and $1 \mathrm{~J} / \mathrm{cm}^{2}$ UVA) were very 
similar to those with 6-TG, including the production of damage, recognised by hOGG1, following treatment with Aza, but in the absence of UVA (Figure 1B). We thereby demonstrated the formation of a significant $(\mathrm{P}<0.0001)$ levels of ALS and 8oxodG in cells treated with 6-TG and UVA (Figure 1A), and likewise for Aza plus UVA (Figure 1B).

We were also able to demonstrate a clear dose-response in 8-oxodG formation, with increasing concentration of 6-TG used (Figure 2). As before, use of hOGG1 in the comet assay revealed greater levels of damage however, in contrast to ALS, levels of 8-oxodG appeared to approach a plateau at the highest doses. In order to investigate whether incorporation of 6-TG into DNA was a pre-requisite for formation of 8-oxodG in DNA, we performed the same experiment, as above, only using serum starved cells. Previous studies have shown that serum starvation for $48 \mathrm{hr}$ renders primary skin fibroblasts quiescent, with $>90 \%$ of the cells arrested in $\mathrm{G}_{0} / \mathrm{G}_{1}$ [34], and we confirmed that this was the case for our HDFs (data not shown). Perhaps surprisingly, we still noted formation of significant levels of damage, both 8-oxodG and ALS (Figure 3), although levels of both were significantly decreased $(\mathrm{P}<0.0001)$ compared to proliferating cells (Table 1). In contrast to what we have noted for proliferating cells (above), treatment of serum starved cells with UVA alone, did appear to induce 8-oxodG, although not ALS (Figure 3).

\subsection{Effect of thiopurines and UVA upon cell viability}

The viability of HDFs following the various combinations of treatments was assessed (Figure 4). Treatment of cells with 6-TG and UVA produced a modest, albeit statistically significant $(\mathrm{p}<0.01)$, decrease in cell viability compared to control, unirradiated cells. In the absence of irradiation, 6-TG had no effect upon viability. 
Treatment of cells with Aza or UVA, individually or in combination, had no effect upon viability. 


\section{Discussion}

There is growing in vitro evidence for the formation of ROS [23] and oxidative stress being induced by 6-TG and UVA, which includes evidence for protein oxidation (PCNA specifically [35]). It might therefore be expected that DNA will also be oxidised. This is the first report to directly demonstrate the formation of significant levels of oxidatively damaged DNA, both ALS and 8-oxodG, in cells treated with biologically relevant doses of 6-TG and UVA. This is an important advance on previous reports, which either simply implied the formation of oxidatively damaged cellular DNA [23], or reported the formation of novel DNA lesions (e.g. $\mathrm{G}^{\mathrm{SO} 3}$ ), but did not demonstrate their presence in the DNA of treated cells [24], and have largely overlooked the formation of other DNA products induced by ${ }^{1} \mathrm{O}_{2}[36]$.

Based upon its substrate specificity, it is important to be circumspect when attributing hOGG1-sensitive sites solely to 8 -oxodG. For example, two ${ }^{\circ} \mathrm{OH}$-induced products, 2,6-diamino-4-hydroxy-formamidopyrimidine (FapyGua) and, to a much lesser extent, 4,6-diamino-5-formamidopyrimidine (FapyAde), are amongst the substrates for hOGG1 [37]. However, under our experimental conditions, we are confident that the majority of the hOGG1-sensitive sites are indeed 8-oxodG. Justification for this derives from: (i) ROS generated by UVA is predominantly ${ }^{1} \mathrm{O}_{2}$ (85\%), rather than ${ }^{\circ} \mathrm{OH}(15 \%)$ [14]; (ii) existing work on Aza/6-TG and UVA report ${ }^{1} \mathrm{O}_{2}$ to be the major damaging species [35], presumably over and above that seen with UVA alone; plus (iii) guanine is sole the target for ${ }^{1} \mathrm{O}_{2}$ under neutral aqueous conditions [38]; and (iv) 8-oxodG is the predominant form of DNA damage induced by ${ }^{1} \mathrm{O}_{2}[39]$.

In this study, approximately $50 \%$ of the total damage is 8 -oxodG, the remainder being ALS (which includes SSB and AP sites). Whether ${ }^{1} \mathrm{O}_{2}$ can generate 
SSB and AP sites in cellular DNA has been the subject of some debate. However, a thorough study by Ravanat et al. [40], using a pure ${ }^{1} \mathrm{O}_{2}$-generating system, concluded that ${ }^{1} \mathrm{O}_{2}$ does not produce significant levels of SSB or AP sites. The authors went on to suggest that low levels of ALS are the consequence of subsequent oxidation of 8oxodG to products such as imidazolone, oxazolone [41] and oxaluric acid [42], some of which are alkali-labile [32]. It is also possible that SSB arise, transiently, from endogenous, cellular repair activity towards DNA damage [40] e.g. hOGG1. In contrast to the water soluble, thermolabile endoperoxide-derived ${ }^{1} \mathrm{O}_{2}$ used in the above study, which is generated in cell culture medium, we would argue that 6-TGderived ${ }^{1} \mathrm{O}_{2}$ is generated in relative close proximity to DNA. On this basis, levels of 8-oxodG and its secondary oxidation products would be expected to be much greater compared to the system used by Ravanat et al. [40], and hence could account for the greater number of ALS we report. It has been reported that performing unwinding and electrophoresis at $\mathrm{pH} \sim 12$ versus $\mathrm{pH}>13$ can discriminate between agents which induce SSB alone, and those that induce ALS [43]. Repeating the above experiment, comparing comet conditions at $\mathrm{pH} \sim 12$ to $\mathrm{pH}>13$ indicated minimal SSB formation following treatment of cells with 6-TG plus UVA (data not shown), although this result might be viewed with caution, as normal and oxidised AP sites are readily converted to SSB under mildly alkali conditions. Similar levels, and ratio of 8-oxodG to ALS, of damage were also noted in cells treated with Aza, confirming the ability of HDFs to metabolise Aza.

CPD and pyrimidine (6-4) pyrimidone photoproducts are widely considered to be the lesions responsible for mutagenicity of solar UVR [44], an argument strengthened by the growing evidence that pure UVA can also induce CPD $[13,33]$. On this basis, CPD formed during the treatment of cells with Aza in conjunction with 
UVA, could be the underlying cause of the mutagenicity of this treatment. However, we demonstrate that, dose-for-dose, minimal levels of CPD are induced following UVA alone, compared to 8-oxodG and ALS in UVA + 6-TG-treated cells.

The results for the cells treated with 6-TG (or indeed Aza), but not UVA, and assayed by hOGG1 comet do not seem entirely straight forward, as they showed a significant, $50 \%$ increase in damage, compared their to corresponding control. In the absence of hOGG1, no ALS were detected. This suggests that, either (i) 8-oxodG, specifically, is formed due to ambient, white light exposure, or, (ii) to some extent, 6TG, or derivatives thereof, in DNA is a substrate for hOGG1, at the particular concentration of enzyme and incubation period used. The former also relies upon the formation of 8-oxodG, in the absence of ALS which if, as for UVA, the damaging species is ${ }^{1} \mathrm{O}_{2}$, would seem unlikely, furthermore we have been unable to demonstrate 8-oxodG formation in 6-TG treated cells irradiated with white light (data not shown). The second hypothesis adds 6-TG to the substrate repertoire of hOGG1 which has been reported to be 8-oxoGua, methyl-2,6-diamino-4-hydroxy-5formamidopyrimidine and FapyGua, preferentially when paired opposite cytosine (Cyt) [45], the latter adding a further level of specificity [37]. Furthermore, 6-TG is structurally very different from 8-oxodG, so removal of this modified base by hOGG1 might be considered unlikely. However, recently it has been reported that hOGG1 also has some activity towards 8-thio-2'-dG, 7-methyl-8-oxo-2'-dG and 7-deaza-2'$\mathrm{dG}$ in double-stranded oligonucleotides [46]. Additionally, it would appear that hOGG1 can catalyse $N$-glycosidic bond cleavage of the human cytomegalovirus inhibitor 2-bromo-5,6-dichloro-1-(-D-ribofuranosyl)benzimidazole, and its 2-chloro homologue, 2,5,6-trichloro-1-(-D-ribofuranosyl)benzimidazole [47]. Whilst this latter activity is towards free nucleosides, these two reports nevertheless raise the possibility 
that the repertoire for hOGG1 may be greater than previously thought, and might include 6-TG, especially if high levels of incorporation have occurred. In DNA, 6-TG is incorporated as if it were guanine, opposite Cyt, which is at least a base pairing that matches the known repertoire of hOGG1. It should be considered that $\sim 1$ in $10^{4}$ to 1 in $10^{5}$ DNA 6-TG can be methylated to 6-methyl-thioguanine [7] and hence may also represent a possible substrate for hOGG1. However, structurally 6-me-TG is even less like 8-oxodG and, after replication, mis-pairs with thymine. Further, indirect evidence that 6-TG in DNA might be a substrate for hOGG1 comes from the results with non-proliferating cells. In this experiment, the CSI + 6-TG treated cells do not show any increase in percentage tail DNA, unlike the corresponding treatment in proliferating cells. This is explained by 6-TG not having been incorporated into DNA, and is hence not available as a substrate for hOGG1. The possibility of hOGG1 having a broader substrate specificity warrants further investigation for, at present, it is not known whether or not hOGG1 recognises 6-TG, its methylated equivalent, or indeed its putative oxidation products, such as $\mathrm{G}^{\mathrm{SO}}$.

Levels of 8-oxodG and ALS increased with increasing dose of 6-TG, in conjunction with UVA. Damage was induced with doses of 6-TG as low as $0.2 \mu \mathrm{M}$ and, whilst 8-oxodG levels appeared to plateau at around $1 \mu \mathrm{M}$, this was not the case for ALS which increased further at $5 \mu \mathrm{M}$. Explanation for this may be that the amount of substrate has saturated available enzyme, or that 6-TG incorporation plateaus at high 6-TG concentrations.

Incorporation of thiopurine derivatives into DNA is thought to be a prerequisite for their clinical effectiveness, whereas inhibition of de novo purine synthesis has a less significant role [7]. In contrast, our data with quiescent cells indicates that some damage still occurs, albeit at significantly lower levels than in 
proliferating cells. This would imply that, even in non-proliferating cells, there is still nucleobase, and hence 6-TG, uptake and metabolism to 2'-deoxyribonucleotides, even if no incorporation into DNA occurs. On this basis, we surmise that it is still possible for the 6-TG metabolite, 6-TdGTP, to absorb UVA and form ${ }^{1} \mathrm{O}_{2}$ which can subsequently damage DNA. As discussed above, generation of ${ }^{1} \mathrm{O}_{2}$ away from the close proximity of DNA would be expected to produce lower levels of damage than those derived from photosensitised formation from 6-TG in DNA, hence we do see damage in non-proliferating cells, but much less than in actively dividing cells. Another phenomenon observed was that, in contrast to actively dividing cells, irradiation of non-proliferating HDFs with UVA alone, resulted in a modest, but significant, increase in 8-oxodG, although not ALS. This implies a greater sensitivity to UVA in non-proliferating, compared to proliferating cells.

In our previous work, we reported a significant association between Aza treatment and urinary 8 -oxodG $(P=0.02)$ in renal transplant patients. The current understanding is that the most likely and logical source of urinary 8-oxodG is from sanitisation of the 2'-deoxyribonucleotide pool [48], this and the discussion in the paragraph above raise the question, is the dNTP pool a target for 6-TG induced oxidation? It seems highly likely that 6-TG is present in the dNTP pool as, for incorporation into DNA to occur, 6-TG must be converted into 6-TdGTP which is then a substrate for DNA polymerases [49]. There is also good evidence demonstrating that 6-TG does not have to be in the context of DNA in order to generate ${ }^{1} \mathrm{O}_{2}$ upon UVA irradiation. Aqueous solutions of both 6-TG and its corresponding 2'-deoxyribonucleoside can autooxidise, following UVA irradiation and subsequent generation of ${ }^{1} \mathrm{O}_{2}$ [24]. There is precedent for an agent, which is normally thought to target DNA, to also exert a similar effect via modification of free 
2'-deoxyribonucleotides and incorporation into DNA [50]. It is therefore plausible that ${ }^{1} \mathrm{O}_{2}$, generated in the dNTP pool, modifies dGTP, which is a substrate for Nudix hydrolases, such as NUDT1 [51]. This therefore represents a plausible mechanism for the elevated levels of urinary 8-oxodG we noted previously [26].

Perhaps surprisingly, treatment with 6-TG and UVA, but not Aza, lead to a small but significant decrease in viability, despite both treatments inducing approximately the same amount of damage. It is possible that, the additional metabolic steps required to convert Aza to TdGTP, limit the amount of incorporation in our experimental system, compared to incubation with 6-TG, but the amount of incorporation that does occur is sufficient to generate levels of 8-oxodG seen with 6TG.

Combined, the data presented here, along with other reports emerging in the literature, strongly suggest that Aza-derived oxidative stress, and damage to DNA specifically, will occur in the skin of patients on Aza, following even low level UVA exposure. This is likely to be an important risk factor for non-melanoma skin cancer in these patients. However, as it would appear that oxidative stress is not involved in the therapeutic effects of Aza, which occur in the absence of UVA exposure, therefore intercepting ROS production in the skin could be a viable route by which the risk of this side effect may be minimised. 


\section{Acknowledgements}

MSC and MDE are partners of ECNIS (Environmental Cancer Risk, Nutrition and Individual Susceptibility), a network of excellence operating within the European Union $6^{\text {th }}$ Framework Program, Priority 5:"Food Quality and Safety" (Contract No 513943). 


\section{References}

[1] J. Aarbakke, G. Janka-Schaub and G.B. Elion Thiopurine biology and pharmacology, Trends Pharmacol Sci 18 (1997) 3-7.

[2] R. Weinshilboum Thiopurine pharmacogenetics: clinical and molecular studies of thiopurine methyltransferase, Drug Metab Dispos 29 (2001) 601605.

[3] J. Bohon and C.R. de los Santos Effect of 6-thioguanine on the stability of duplex DNA, Nucleic Acids Res 33 (2005) 2880-2886.

[4] G.A. Lepage Basic biochemical effects and mechanism of action of 6thioguanine, Cancer Res 23 (1963) 1202-1206.

[5] S.H. Lee and A.C. Sartorelli The effects of inhibitors of DNA biosynthesis on the cytotoxicity of 6-thioguanine, Cancer Biochem Biophys 5 (1981) 189-194.

[6] C.R. Fairchild, J. Maybaum and K.A. Kennedy Concurrent unilateral chromatid damage and DNA strand breakage in response to 6-thioguanine treatment, Biochem Pharmacol 35 (1986) 3533-3541.

[7] P. Karran and N. Attard Thiopurines in current medical practice: molecular mechanisms and contributions to therapy-related cancer, Nat Rev Cancer 8 (2008) 24-36.

[8] S. Euvrard, J. Kanitakis and A. Claudy Skin cancers after organ transplantation, N Engl J Med 348 (2003) 1681-1691.

[9] C. Ulrich and E. Stockfleth Azathioprine, UV light, and skin cancer in organ transplant patients--do we have an answer?, Nephrol Dial Transplant 22 (2007) 1027-1029.

[10] J.S. Maddox and K. Soltani Risk of nonmelanoma skin cancer with azathioprine use, Inflamm Bowel Dis (2008). 
[11] J.A. Parrish Immunosuppression, skin cancer, and ultraviolet A radiation, $\mathrm{N}$ Engl J Med 353 (2005) 2712-2713.

[12] V.E. Reeve, G.E. Greenoak, C.H. Gallagher, P.J. Canfield and F.J. Wilkinson Effect of immunosuppressive agents and sunscreens on UV carcinogenesis in the hairless mouse, Aust J Exp Biol Med Sci 63 ( Pt 6) (1985) 655-665.

[13] S. Mouret, C. Baudouin, M. Charveron, A. Favier, J. Cadet and T. Douki Cyclobutane pyrimidine dimers are predominant DNA lesions in whole human skin exposed to UVA radiation, Proc Natl Acad Sci U S A 103 (2006) 1376513770.

[14] J. Cadet, E. Sage and T. Douki Ultraviolet radiation-mediated damage to cellular DNA, Mutat Res 571 (2005) 3-17.

[15] R.M. Tyrrell and V.E. Reeve Potential protection of skin by acute UVA irradiation--from cellular to animal models, Prog Biophys Mol Biol 92 (2006) 86-91.

[16] S.P. Hussain, L.J. Hofseth and C.C. Harris Radical causes of cancer, Nat Rev Cancer 3 (2003) 276-285.

[17] M.S. Cooke, M.D. Evans, M. Dizdaroglu and J. Lunec Oxidative DNA damage: mechanisms, mutation, and disease, FASEB J 17 (2003) 1195-1214.

[18] M.D. Evans and M.S. Cooke Factors contributing to the outcome of oxidative damage to nucleic acids, Bioessays 26 (2004) 533-542.

[19] H.S. Black Reassessment of a free radical theory of cancer with emphasis on ultraviolet carcinogenesis, Integr Cancer Ther 3 (2004) 279-293.

[20] C. Nishigori Cellular aspects of photocarcinogenesis, Photochem Photobiol Sci 5 (2006) 208-214. 
[21] C. Nishigori, Y. Hattori and S. Toyokuni Role of reactive oxygen species in skin carcinogenesis, Antioxid Redox Signal 6 (2004) 561-570.

[22] D.R. Bickers and M. Athar Oxidative stress in the pathogenesis of skin disease, J Invest Dermatol 126 (2006) 2565-2575.

[23] P. O'Donovan, C.M. Perrett, X. Zhang, B. Montaner, Y.Z. Xu, C.A. Harwood, J.M. McGregor, S.L. Walker, F. Hanaoka and P. Karran Azathioprine and UVA light generate mutagenic oxidative DNA damage, Science 309 (2005) $1871-1874$.

[24] X. Zhang, G. Jeffs, X. Ren, P. O'Donovan, B. Montaner, C.M. Perrett, P. Karran and Y.Z. Xu Novel DNA lesions generated by the interaction between therapeutic thiopurines and UVA light, DNA Repair (Amst) 6 (2007) 344-354.

[25] G.E. Kelly, W.D. Meikle and D.E. Moore Enhancement of UV-induced skin carcinogenesis by azathioprine: role of photochemical sensitisation, Photochem Photobiol 49 (1989) 59-65.

[26] M.S. Cooke, J.E. Osborne, R. Singh, V. Mistry, P.B. Farmer, M.D. Evans and P.E. Hutchinson Evidence that oxidative stress is a risk factor for the development of squamous cell carcinoma in renal transplant patients, Free Radic Biol Med 43 (2007) 1328-1334.

[27] T.L. Duarte, G.M. Almeida and G.D. Jones Investigation of the role of extracellular $\mathrm{H} 2 \mathrm{O} 2$ and transition metal ions in the genotoxic action of ascorbic acid in cell culture models, Toxicol Lett 170 (2007) 57-65.

[28] Y.T. Wu, C. Shen, J. Yin, J.P. Yu and Q. Meng Azathioprine hepatotoxicity and the protective effect of liquorice and glycyrrhizic acid, Phytother Res 20 (2006) 640-645. 
[29] C.C. Smith, M.R. O'Donovan and E.A. Martin hOGG1 recognizes oxidative damage using the comet assay with greater specificity than FPG or ENDOIII, Mutagenesis 21 (2006) 185-190.

[30] E. Horvathova, D. Slamenova, L. Hlincikova, T.K. Mandal, A. Gabelova and A.R. Collins The nature and origin of DNA single-strand breaks determined with the comet assay, Mutat Res 409 (1998) 163-171.

[31] J.R. Sparrow, J. Zhou and B. Cai DNA is a target of the photodynamic effects elicited in A2E-laden RPE by blue-light illumination, Invest Ophthalmol Vis Sci 44 (2003) 2245-2251.

[32] J. Cadet, A.G. Bourdat, C. D'Ham, V. Duarte, D. Gasparutto, A. Romieu and J.L. Ravanat Oxidative base damage to DNA: specificity of base excision repair enzymes, Mutat Res 462 (2000) 121-128.

[33] P.J. Rochette, J.P. Therrien, R. Drouin, D. Perdiz, N. Bastien, E.A. Drobetsky and E. Sage UVA-induced cyclobutane pyrimidine dimers form predominantly at thymine-thymine dipyrimidines and correlate with the mutation spectrum in rodent cells, Nucleic Acids Res 31 (2003) 2786-2794.

[34] F.R. Danesh, M. Ye, S. Salmi, M. Lapointe and D. Batlle Temporal profile of serum-induced S-phase entry and retinoblastoma protein phosphorylation in human skin fibroblasts, Kidney Int 56 (1999) 1282-1285.

[35] B. Montaner, P. O'Donovan, O. Reelfs, C.M. Perrett, X. Zhang, Y.Z. Xu, X. Ren, P. Macpherson, D. Frith and P. Karran Reactive oxygen-mediated damage to a human DNA replication and repair protein, EMBO Rep 8 (2007) 1074-1079. 
[36] J.L. Ravanat, P. Di Mascio, G.R. Martinez, M.H. Medeiros and J. Cadet Singlet oxygen induces oxidation of cellular DNA, J Biol Chem 275 (2000) 40601-40604.

[37] N. Krishnamurthy, K. Haraguchi, M.M. Greenberg and S.S. David Efficient removal of formamidopyrimidines by 8-oxoguanine glycosylases, Biochemistry 47 (2008) 1043-1050.

[38] J.-L. Ravanat, C. Saint-Pierre, P. Di Mascio, G.R. Martinez, M.H.G. Medeiros, J. Cadet and 3702-3709. Damage to isolated DNA mediated by singlet oxygen, Helv. Chim. Acta 84 (2001) 3702-3709. .

[39] B. Epe DNA damage induced by photosensitisation, in: B. Halliwell and O.I. Aruoma (Eds.), DNA and free radicals, Ellis Horwood, New York, 1993, pp. 41-65.

[40] J.L. Ravanat, S. Sauvaigo, S. Caillat, G.R. Martinez, M.H. Medeiros, P. Di Mascio, A. Favier and J. Cadet Singlet oxygen-mediated damage to cellular DNA determined by the comet assay associated with DNA repair enzymes, Biol Chem 385 (2004) 17-20.

[41] G.R. Martinez, M.H. Medeiros, J.L. Ravanat, J. Cadet and P. Di Mascio [180]-labeled singlet oxygen as a tool for mechanistic studies of 8-oxo-7,8dihydroguanine oxidative damage: detection of spiroiminodihydantoin, imidazolone and oxazolone derivatives, Biol Chem 383 (2002) 607-617.

[42] V. Duarte, D. Gasparutto, M. Jaquinod, J. Ravanat and J. Cadet Repair and mutagenic potential of oxaluric acid, a major product of singlet oxygenmediated oxidation of 8-oxo-7,8-dihydroguanine, Chem Res Toxicol 14 (2001) 46-53. 
[43] A.R. Collins, A.A. Oscoz, G. Brunborg, I. Gaivao, L. Giovannelli, M. Kruszewski, C.C. Smith and R. Stetina The comet assay: topical issues, Mutagenesis 23 (2008) 143-151.

[44] A. Besaratinia, S.I. Kim and G.P. Pfeifer Rapid repair of UVA-induced oxidized purines and persistence of UVB-induced dipyrimidine lesions determine the mutagenicity of sunlight in mouse cells, FASEB J (2008).

[45] S.S. David and S.D. Williams Chemistry of glycosylases and endonucleases involved in base-excision repair, Chem Rev 98 (1998) 1221-1262.

[46] M.L. Hamm, T.J. Gill, S.C. Nicolson and M.R. Summers Substrate specificity of Fpg (MutM) and hOGG1, two repair glycosylases, J Am Chem Soc 129 (2007) 7724-7725.

[47] P.L. Lorenzi, C.P. Landowski, A. Brancale, X. Song, L.B. Townsend, J.C. Drach and G.L. Amidon N-methylpurine DNA glycosylase and 8-oxoguanine dna glycosylase metabolize the antiviral nucleoside 2-bromo-5,6-dichloro-1(beta-D-ribofuranosyl)benzimidazole, Drug Metab Dispos 34 (2006) 10701077.

[48] M.S. Cooke, R. Olinski and S. Loft Measurement and meaning of oxidatively modified DNA lesions in urine, Cancer Epidemiol Biomarkers Prev 17 (2008) 3-14.

[49] D.J. Warren, A. Andersen and L. Slordal Quantitation of 6-thioguanine residues in peripheral blood leukocyte DNA obtained from patients receiving 6-mercaptopurine-based maintenance therapy, Cancer Res 55 (1995) 16701674.

[50] A. Arecco, B.J. Mun and C.K. Mathews Deoxyribonucleotide pools as targets for mutagenesis by N-methyl-N-nitrosourea, Mutat Res 200 (1988) 165-175. 
[51] T. Tsuzuki, Y. Nakatsu and Y. Nakabeppu Significance of error-avoiding mechanisms for oxidative DNA damage in carcinogenesis, Cancer Sci 98 (2007) 465-470. 
Table I. Comparison of levels of damage, induced by 6-TG and UVA, in quiescent versus proliferating human diploid fibroblasts (HDFs).

\begin{tabular}{l|cc}
\hline \multicolumn{1}{c|}{ Damage (\% tail DNA) } & \multicolumn{2}{|c}{ Treatment } \\
\hline & Quiescent & Proliferating \\
& HDFs & HDFs \\
\hline 8-oxodG (mean +/- SEM) & $26.4(1.8)$ & $52.5(1.9)^{* * *}$ \\
ALS (mean +/- SEM) & $14.8(0.9)$ & $25.3(1.3)^{* * *}$ \\
\hline$* * * \mathrm{P}<0.0001$ & &
\end{tabular}

$* * * \mathrm{P}<0.0001$ 


\section{Figure legends}

Fig. 1A. Production of oxidatively damaged DNA in HDFs treated with combinations of UVA $\left(1 \mathrm{~J} / \mathrm{cm}^{2}\right)$ and 6-TG $(1 \mu \mathrm{M})$, or Fig. 1B. UVA $\left(1 \mathrm{~J} / \mathrm{cm}^{2}\right)$ and Aza $(1 \mu \mathrm{M})$. DNA damage analysis was by the alkaline comet assay with and without hOGG1, representing both 8-oxodG and ALS, and ALS alone, respectively. In addition, Fig 1A. shows levels of cyclobutane pyrimidine dimers, following $1 \mathrm{~J} / \mathrm{cm}^{2}$ UVA irradiation, determined by the T4 endoV comet assay. Results are the mean (+/) SEM of four individual determinations. $* * \mathrm{P}=0.002$, *** $\mathrm{P}<0.0001$, compared to corresponding control sham irradiated (CSI) sample, in the absence of 6-TG (with or without hOGG1, or T4 endoV, treatment), unless otherwise indicated.

Fig. 2. Dose-response effect of 6-TG treatment, in conjunction with UVA irradiation $\left(1 \mathrm{~J} / \mathrm{cm}^{2}\right)$, on the production of oxidatively damaged DNA in HDFs. DNA damage analysis was by the alkaline comet assay with (open bars) and without (hatched bars) hOGG1, representing both 8-oxodG and ALS, and ALS alone, respectively. Results are the mean (+/-) SEM of four individual determinations. $* * * \mathrm{P}<0.0001$, compared to corresponding control sham irradiated (CSI) sample, in the absence of 6-TG (with or without hOGG1 treatment).

Fig. 3. Effect of treatment with 6-TG $(1 \mu \mathrm{M})$ and UVA $\left(1 \mathrm{~J} / \mathrm{cm}^{2}\right)$ upon the production of oxidatively damaged DNA in HDFs rendered quiescent by serum starvation. DNA damage analysis was by the alkaline comet with (open bars) and without (hatched bars) hOGG1, representing 8-oxodG and ALS, respectively. Results are the mean (+/-) SEM of four individual determinations. $* * * \mathrm{P}<0.0001$; ** $\mathrm{P}<0.01$, compared 
to corresponding control sham irradiated (CSI) sample, in the absence of 6-TG (with or without hOGG1 treatment), unless otherwise indicated.

Fig. 4. Effect of treatment with 6-TG $(1 \mu \mathrm{M})$, or Aza $(1 \mu \mathrm{M})$, plus UVA $\left(1 \mathrm{~J} / \mathrm{cm}^{2}\right)$ upon cell death in HDFs. Cell viability was determined $48 \mathrm{hr}$ after treatment and reintroduction of 6-TG-free medium. $* * \mathrm{P}<0.01$, compared to 6-TG treated, control sham irradiated (CSI) cells. 
1 (A)

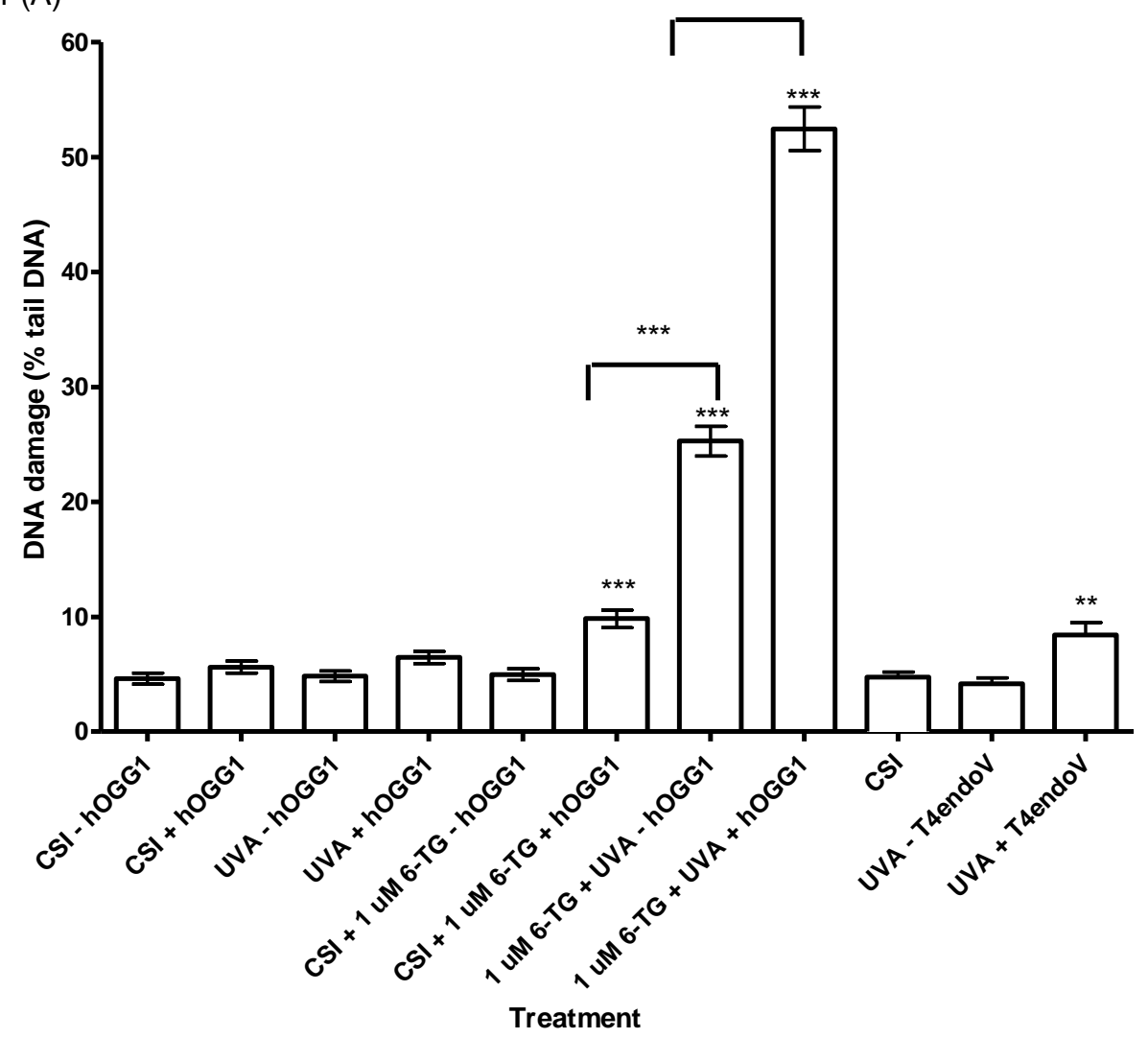

1 (B)

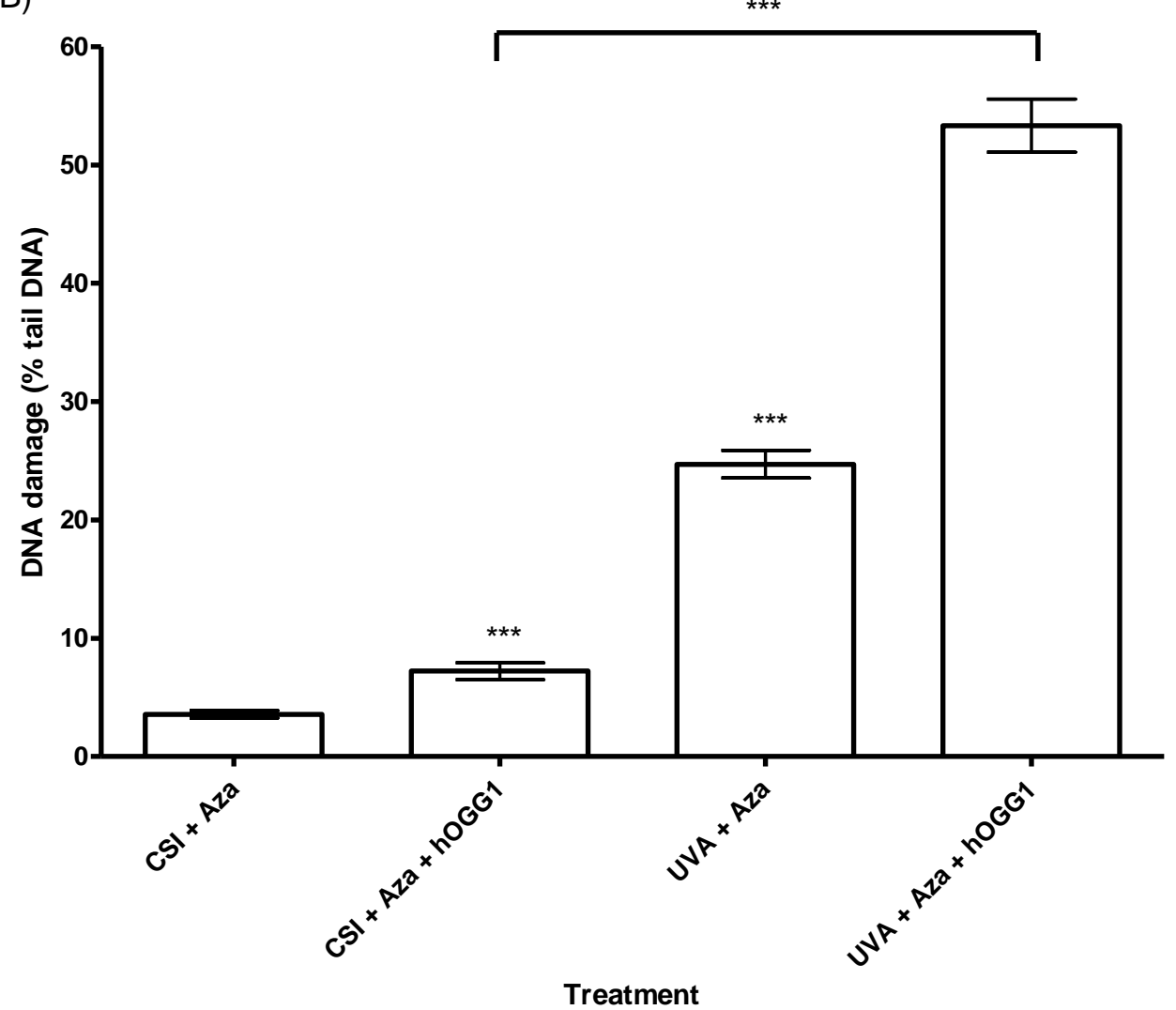



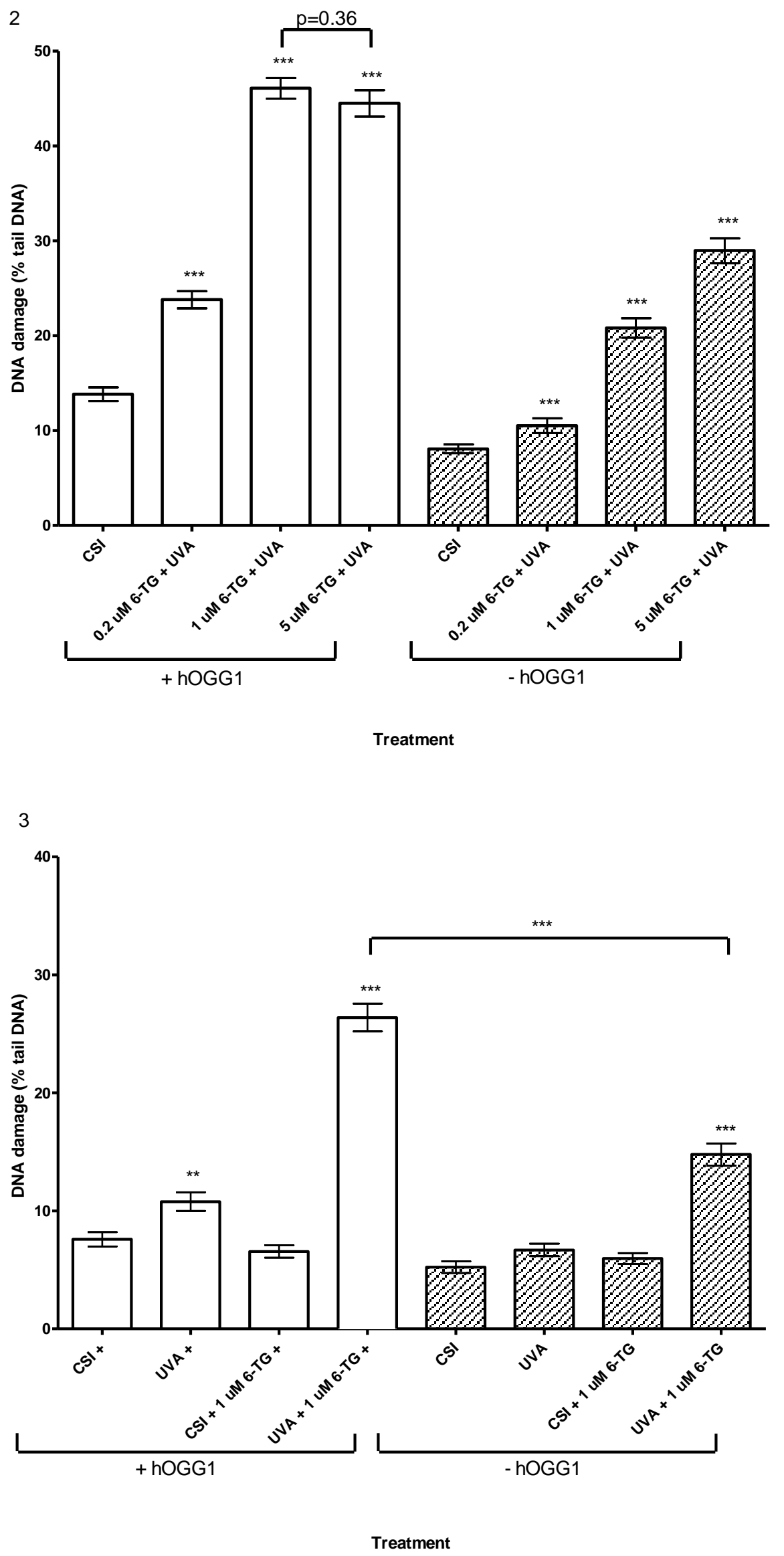


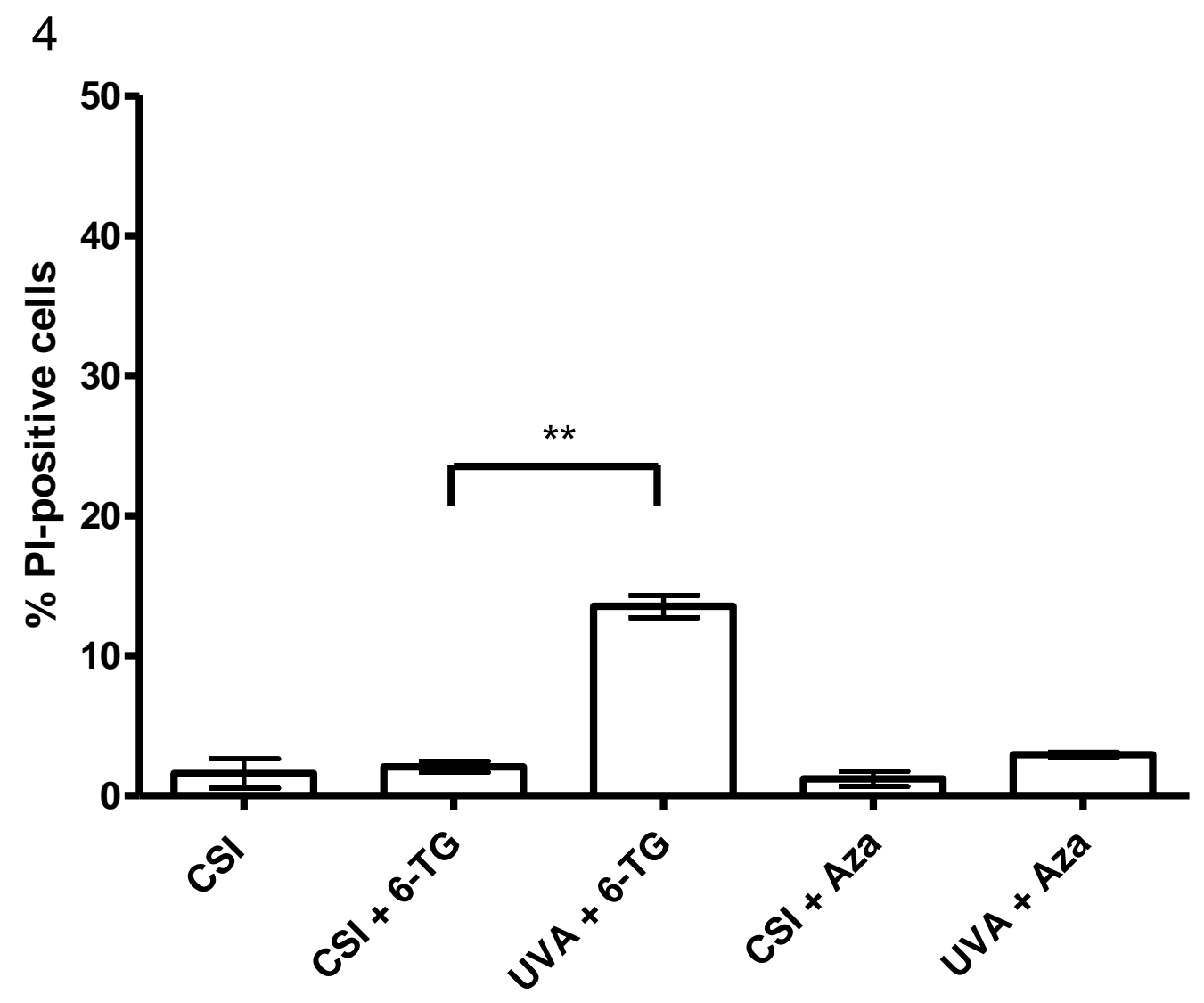

Treatment 